\title{
A Sequential Monte Carlo Method for Bayesian Face Recognition
}

\author{
Atsushi Matsui ${ }^{1,2}$, Simon Clippingdale ${ }^{1}$, \\ and Takashi Matsumoto ${ }^{2}$ \\ ${ }^{1}$ Science \& Technical Research Laboratories, \\ NHK (Japan Broadcasting Corporation), \\ 1-10-11 Kinuta, Setagaya-ku, Tokyo 157-8510 Japan \\ ${ }^{2}$ Dept. of Electrical Engineering \& Bioscience, Waseda University, \\ 3-4-1 Okubo, Shinjuku-ku, Tokyo 169-8555 Japan
}

\begin{abstract}
This paper proposes a Sequential Monte Carlo (SMC) learning algorithm for Bayesian probability distributions that describe model parameters in a video face recognition system based on deformable template matching. The new algorithm achieves significantly improved robustness of recognition against facial expressions and speech movements by comparison with a baseline batch MCMC (Markov Chain Monte Carlo) algorithm, at no additional computational cost. Experimental results demonstrate the effectiveness and computational efficiency of the new algorithm.
\end{abstract}

\section{Introduction}

Human faces in broadcast video exhibit substantial variation in position, size, head pose, facial expression and so on, forcing face recognition systems for video indexing to incorporate flexibility in the database and/or matching algorithms used. The authors have introduced a prototype recognition system[1] [2] which uses deformable template matching and is based on the Elastic Graph Matching $\operatorname{method}[3$. Although this sytem can absorb a certain amount of facial deformation due to expressions and speech movements, recognition errors can occur for larger deformations, and additionally there are a number of system hyperparameters which are set in a heuristic fashion.

In this work, we introduce an online learning algorithm for Bayesian posterior probabilities describing faces in video input sequences, which uses a Sequential Monte Carlo (SMC) method [4] [5] to perform integrations over a sequence of combined spaces of face model parameters and system hyperparameters. We show that this SMC approach successfully adapts the parameters associated with deformations of each face model, and significantly reduces recognition errors on a video test set showing individuals talking, relative to a baseline batch MCMC (Markov Chain Monte Carlo) algorithm 6] 7]. However, it does so at increased computational cost. We then introduce a modification at the resampling stage of the algorithm that restores computational efficiency (to somewhat better than 
that of the baseline MCMC algorithm) without sacrificing the gain in recognition performance achieved by the SMC algorithm.

In section 2 we briefly review the deformable template matching procedure and similarity function used in our original system. In section 3 we introduce the online learning approach where a new likelihood function is proposed, defined in terms of a mixture of von Mises-Fisher distributions, and show how the most probable model can be estimated together with distributions of system parameters. In section 4 we describe the details of the SMC algorithm with experimental results, and introduce the modification of the resampling stage that boosts computational efficiency. The paper concludes with a discussion of the results and possible directions for further work.

\section{Deformable Template Matching}

The deformable templates used in our original system [1] are constructed from face images of target individuals at multiple poses, labeled with feature point positions. Each template consists of the normalized coordinates of $M=9$ feature points, $\boldsymbol{x}^{A}=\left\{\boldsymbol{x}_{1}^{A}, \ldots, \boldsymbol{x}_{M}^{A}\right\}$, together with features $\boldsymbol{c}^{A}$ computed by convolutions with Gabor wavelets at each of the feature points. The Gabor wavelet at resolution $r$ and orientation $n$ is a complex exponential grating patch with a 2-D Gaussian envelope:

$$
g_{n}^{r}(\boldsymbol{x})=\frac{k_{r}^{2}}{\sigma^{2}} e^{-\frac{k_{r}^{2} \| \boldsymbol{x}_{\|}^{2}}{2 \sigma^{2}}} \times\left[e^{i\left(\boldsymbol{k}_{n}^{r}\right)^{T} \boldsymbol{x}}-e^{-\frac{\sigma^{2}}{2}}\right], \quad \boldsymbol{k}_{n}^{r}=k_{r}\left(\begin{array}{c}
\cos \left(\frac{n \pi}{N_{\text {orn }}}\right) \\
\sin \left(\frac{n \pi}{N_{\text {orn }}}\right)
\end{array}\right),
$$

for $N_{\text {orn }}=8$ orientations and $R=5$ resolutions. This data representation is similar to that used in the Elastic Graph Matching system 3 for face recognition in static images, but the chosen feature points differ, as do the parameters of the Gabor wavelets. The original scheme[1] 2] applies templates to input video frames and deforms them by shifting the feature points so as to maximize the similarity to the Gabor features in the template. It then computes an overall match score for each deformed template, incorporating a feature similarity term and a penalty related to the deformation as follows:

$$
S_{A, B}^{r}=1-\alpha_{f}\left(1-\frac{\left\langle\boldsymbol{c}_{r}^{A}, \boldsymbol{c}_{r}^{B}\right\rangle}{\left|\boldsymbol{c}_{r}^{A}\right|\left|\boldsymbol{c}_{r}^{B}\right|}\right)-\alpha_{s} \frac{\sqrt{E_{A, B}}}{\lambda_{r}},
$$

where $A$ denotes the undeformed template and $B$ the deformed feature points on the image; $\boldsymbol{c}^{A}$ and $\boldsymbol{c}^{B}$ are feature vectors of Gabor wavelet coefficients, respectively from the template and measured at the deformed feature point positions $\boldsymbol{x}^{B}$ on the image; $E_{A, B}$ is the deformation energy between the feature points $\boldsymbol{x}^{A}$ in the template and the deformed feature points $\boldsymbol{x}^{B}$ on the image, up to a dilation, rotation and shift; $\alpha_{f}$ and $\alpha_{s}$ are weights for the feature similarity and spatial deformation terms respectively; and $\lambda_{r}=2 \pi / k_{r}$ is the modulation wavelength of the Gabor wavelet at resolution $r$. In the sequel we will often omit the $A$ and $B$ superscripts where the meaning is clear. 


\section{Online Bayesian Learning}

Optimizing a target function with penalty terms can be considered as maximizing the Bayesian posterior probabilities of parameters [9]. From the same viewpoint, the most probable model describing faces in input video is defined by the mode of posterior distributions of face models. Though the optimum set of parameters in some case may yield satisfactory performance in other cases, problems can arise if the target probability distribution takes on a more complex form. In general, finding the global maximum of a target function is difficult, and prone to falling into local maxima. Moreover, the optimal parameter set obviously depends on unknown input data.

In this paper, instead of searching for the mode, we introduce an online learning algorithm to estimate both the peak and tails of probability distributions of parameters.

\subsection{Likelihood Function}

Consider the situation where data is given as a video sequence. Let $y_{n}$ be image data at the $n$th frame and let $y_{1: n}=\left\{y_{1}, y_{2} . ., y_{n}\right\}$ be the image data set up to the current frame. Recall that the feature similarity term in equation (2) depends on an innner product between two normalized feature vectors. Therefore it is natural to consider as a likelihood function for this directional data a mixture of von Mises-Fisher distributions 8 :

$$
\begin{aligned}
P\left(y_{n} \mid \boldsymbol{x}_{n}, \beta_{n, 1: R}, \mathcal{H}_{j}\right) & =\frac{1}{R} \sum_{r=1}^{R} \frac{1}{Z_{b}\left(\beta_{n, r}\right)} \exp \left(\beta_{n, r} \frac{\left\langle\boldsymbol{c}_{r}^{A}, \boldsymbol{c}_{r}^{B}\right\rangle}{\left|\boldsymbol{c}_{n, r}^{A}\right|\left|\boldsymbol{c}_{n, r}^{B}\right|}\right), \\
Z_{b}(\beta) & =\frac{(2 \pi)^{\frac{k}{2}} I_{\frac{k}{2}-1}(\beta)}{\beta^{\frac{k}{2}-1}} .
\end{aligned}
$$

where $\boldsymbol{x}_{n}$ represents a set of feature points at the $n$th frame, $\beta_{n, r}$ is a hyperparameter for resolution $r$, and $\mathcal{H}_{j}$ is a face model (hypothesis or template) with identity number $j . I_{p}(\beta)$ is the modified Bessel function, and $k=2 M \times N_{\text {orn }}$.

\subsection{Parameter/Hyperparameter Dynamics}

Suppose that we are provided with a set of feature point locations for the $j$ th template, $\boldsymbol{x}_{j}^{A}$. We assume a Gaussian predictive distribution for $\boldsymbol{x}_{n}$ :

$$
P\left(\boldsymbol{x}_{n} \mid \alpha_{n}, T_{n}, \mathcal{H}_{j}\right)=\frac{1}{Z_{a}\left(\alpha_{n}\right)} \exp \left(-\frac{\alpha_{n}}{2}\left(T_{n}^{-1}\left(\boldsymbol{x}_{n}\right)-\boldsymbol{x}_{j}^{A}\right)^{T} \Lambda_{j}^{-1}\left(T_{n}^{-1}\left(\boldsymbol{x}_{n}\right)-\boldsymbol{x}_{j}^{A}\right)\right),
$$

where $\alpha_{n}$ is a hyperparameter, $\Lambda_{j}$ is the covariance matrix of feature point positions for face model $\mathcal{H}_{j}$, and $Z_{a}\left(\alpha_{n}\right)=(2 \pi)^{M} \sqrt{\operatorname{det} \Lambda_{j} / \alpha_{n}}$ is a normalizing factor.

$T_{n}$ is a rigid linear transformation of the feature point set, consisting of a dilation by a factor $r_{n}$, rotation through an angle $\theta_{n}$, and translation $\left(u_{n}, v_{n}\right)^{T}$. 
It expresses the rigid component of the mapping from the template feature point set $\boldsymbol{x}^{A}$ onto the deformed feature point set $\boldsymbol{x}^{B}$ on the input image plane, leaving the nonrigid deformation.

$$
T_{n}\left(\boldsymbol{x}^{A}\right)=\left[\begin{array}{ccccc}
r_{n} \cos \theta_{n} & -r_{n} \sin \theta_{n} & 0 & \ldots & 0 \\
r_{n} \sin \theta_{n} & r_{n} \cos \theta_{n} & & & \vdots \\
0 & & \ddots & & 0 \\
\vdots & & & & r_{n} \cos \theta_{n}-r_{n} \sin \theta_{n} \\
0 & \ldots & 0 & r_{n} \sin \theta_{n} & r_{n} \cos \theta_{n}
\end{array}\right]\left[\begin{array}{l}
x_{1}^{A} \\
y_{1}^{A} \\
\vdots \\
x_{M}^{A} \\
y_{M}^{A}
\end{array}\right]+\left[\begin{array}{l}
u_{n} \\
v_{n} \\
\vdots \\
u_{n} \\
v_{n}
\end{array}\right]
$$

For simplicity we will use the symbolic notation $T_{n}$ to denote the set of mapping parameters $\left(r_{n}, \theta_{n}, u_{n}, v_{n}\right)$, and $\Theta_{n}$ to denote the set of model parameters $\left(\boldsymbol{x}_{n}, \alpha_{n}, \beta_{n, 1: R}\right)$. The parameters of $T_{n}$ determine the size, position, and in-plane rotation angle of a face region. In this paper, we describe a sequential learning algorithm to estimate probability distributions of these parameters given a sequence of input images.

One way of performing online learning is to consider stochastic updates of the parameters in question. Assuming smooth motion of the target face region, we consider as a recursive update $P\left(T_{n} \mid T_{n-1}\right)$ described by

$$
\begin{aligned}
r_{n}=r_{n-1}+\nu_{r}, & \nu_{r} \sim \mathcal{N}\left(0, \sigma_{r}^{2}\right), \\
\theta_{n}=\theta_{n-1}+\nu_{\theta}, & \nu_{\theta} \sim \mathcal{N}\left(0, \sigma_{\theta}^{2}\right), \\
u_{n}=u_{n-1}+\nu_{x}, & \nu_{x} \sim \mathcal{N}\left(0, \sigma_{x}^{2}\right), \\
v_{n}=v_{n-1}+\nu_{y}, & \nu_{y} \sim \mathcal{N}\left(0, \sigma_{y}^{2}\right) .
\end{aligned}
$$

Similarly, we adopt a transition model for $P\left(\alpha_{n}, \beta_{n, 1: R} \mid \alpha_{n-1}, \beta_{n-1,1: R}\right)$ :

$$
\begin{aligned}
\log \alpha_{n}=\log \alpha_{n-1}+\nu_{\alpha}, & \nu_{\alpha} \sim \mathcal{N}\left(0, \sigma_{\alpha}^{2}\right), \\
\log \beta_{n, r}=\log \beta_{n-1, r}+\nu_{\beta}, & \nu_{\beta} \sim \mathcal{N}\left(0, \sigma_{\beta}^{2}\right),
\end{aligned}
$$

where the log-normal distribution guarantees positivity of the hyperparameters.

\subsection{Posterior Distribution}

Using Bayes' theorem we obtain straightforwardly a recursive formula for $P\left(\mathcal{H}_{j} \mid y_{1: n}\right)$, the posterior distribution of the model $\mathcal{H}_{j}$ given the data:

$$
P\left(\mathcal{H}_{j} \mid y_{1: n}\right)=\frac{P\left(y_{n} \mid y_{1: n-1}, \mathcal{H}_{j}\right) P\left(\mathcal{H}_{j} \mid y_{1: n-1}\right)}{P\left(y_{n} \mid y_{1: n-1}\right)}
$$

where the one-step model marginal likelihood $P\left(y_{n} \mid y_{1: n-1}, \mathcal{H}_{j}\right)$ is given by the following integrals:

$$
P\left(y_{n} \mid y_{1: n-1}, \mathcal{H}_{j}\right)=\int P\left(y_{n} \mid \Theta_{n}, \mathcal{H}_{j}\right) P\left(\Theta_{n}, T_{n} \mid y_{1: n-1}, \mathcal{H}_{j}\right) d\left(\Theta_{n}, T_{n}\right),
$$




$$
\begin{aligned}
P\left(\Theta_{n}, T_{n} \mid y_{1: n-1}, \mathcal{H}_{j}\right)= & \int P\left(\Theta_{n}, T_{n} \mid \Theta_{n-1}, T_{n-1}, \mathcal{H}_{j}\right) \\
& P\left(\Theta_{n-1}, T_{n-1} \mid y_{1: n-1}, \mathcal{H}_{j}\right) d\left(\Theta_{n-1}, T_{n-1}\right) .
\end{aligned}
$$

At each frame, the system outputs the most probable face model, $\mathcal{H}_{M P}^{(n)}$, which attains the maximum value of the posterior distribution of the model given the sequence of input images: $\mathcal{H}_{M P}^{(n)}=\arg \max _{j} P\left(\mathcal{H}_{j} \mid y_{1: n}\right)$.

\section{Sequential Monte Carlo Algorithm}

\subsection{Sequential Importance Sampling}

We cannot typically compute the recursive posterior distribution analytically, because it requires evaluation of the complex high-dimensional integrals in (10) and (11). Instead we apply a Monte Carlo method to estimate the integral numerically. Sequential Monte Carlo requires proposal distributions from which one can draw samples $\left\{\Theta_{n}^{(i)}, T_{n}^{(i)}\right\}_{i=1}^{N_{j}}$ for each model $\mathcal{H}_{j}$ by standard methods. The proposal distribution in this paper will be given by

$$
\begin{aligned}
& \pi\left(\Theta_{n}, T_{n} \mid \mathcal{H}_{j}\right) \\
& \quad=P\left(\boldsymbol{x}_{n} \mid \alpha_{n}, T_{n}, \mathcal{H}_{j}\right) P\left(\alpha_{n} \mid \alpha_{n-1}, \sigma_{\alpha}\right) P\left(\beta_{n, 1: R} \mid \beta_{n-1,1: R}, \sigma_{\beta}\right) P\left(T_{n} \mid T_{n-1}\right)
\end{aligned}
$$

from which one obtains the following approximations:

$$
\begin{aligned}
& P\left(\Theta_{n}, T_{n} \mid y_{1: n-1}, \mathcal{H}_{j}\right) \cong \sum_{i=1}^{N_{j}} \tilde{w}_{n-1}^{(i)} \mid \mathcal{H}_{j} \times \delta\left(\|\left(\Theta_{n}, T_{n}\right)-\left(\Theta_{n}^{(i)}, T_{n}^{(i)}\right)||\right), \\
& P\left(y_{n} \mid y_{1: n-1}, \mathcal{H}_{j}\right)=\int P\left(y_{n} \mid \Theta_{n}, \mathcal{H}_{j}\right) P\left(\Theta_{n}, T_{n} \mid y_{1: n-1}, \mathcal{H}_{j}\right) d\left(\Theta_{n}, T_{n}\right) \\
& \cong \sum_{i=1}^{N_{j}} P\left(y_{n} \mid \Theta_{n}^{(i)}, \mathcal{H}_{j}\right) \times \tilde{w}_{n-1}^{(i)} \mid \mathcal{H}_{j},
\end{aligned}
$$

where the normalized importance weights $\tilde{w}_{n}^{(i)}$ are equal to:

$$
\begin{aligned}
\left.\tilde{w}_{n}^{(i)}\right|_{\mathcal{H}_{j}} & =\frac{\left.w_{n}^{(i)}\right|_{\mathcal{H}_{j}}}{\left.\sum_{k=1}^{N_{\text {persons }}} \sum_{m}^{N_{k}} w_{n}^{(m)}\right|_{\mathcal{H}_{k}}}, \\
\left.w_{n}^{(i)}\right|_{\mathcal{H}_{j}} & =\frac{P\left(y_{n} \mid \Theta_{n}^{(i)}, T_{n}^{(i)}, y_{1: n-1}, \mathcal{H}_{j}\right) P\left(\Theta_{n}^{(i)}, T_{n}^{(i)} \mid \Theta_{n-1}^{(i)}, T_{n-1}\right)}{\pi\left(\Theta_{n}^{(i)}, T_{n}^{(i)} \mid \mathcal{H}_{j}\right)} \times\left. w_{n}^{(i)}\right|_{\mathcal{H}_{j}} \\
& =P\left(y_{n} \mid \Theta_{n}^{(i)}, T_{n}^{(i)}, \mathcal{H}_{j}\right) \times w_{n-1}^{(i)} \mid \mathcal{H}_{j} .
\end{aligned}
$$

In the same way, one can evaluate the sequential model posterior distribution by

$$
\begin{aligned}
P\left(\mathcal{H}_{j} \mid y_{1: n}\right) & =\frac{P\left(y_{n} \mid y_{1: n-1}, \mathcal{H}_{j}\right) P\left(\mathcal{H}_{j} \mid y_{1: n-1}\right)}{\sum_{k=1}^{N_{\text {persons }}} P\left(y_{n} \mid y_{1: n-1}, \mathcal{H}_{k}\right) P\left(\mathcal{H}_{k} \mid y_{1: n-1}\right)} \\
& \cong \frac{\left.P\left(\mathcal{H}_{j} \mid y_{1: n-1}\right) \sum_{i=1}^{N_{j}} \tilde{w}_{n}^{(i)}\right|_{\mathcal{H}_{j}}}{\left.\sum_{k=1}^{N_{\text {persons }}} P\left(\mathcal{H}_{k} \mid y_{1: n-1}\right) \sum_{i=1}^{N_{k}} \tilde{w}_{n}^{(i)}\right|_{\mathcal{H}_{k}}} .
\end{aligned}
$$


Unless there are reasons to do otherwise, we set the initial model probabilities uniformly: $P\left(\mathcal{H}_{j} \mid y_{0}\right)=1 / N_{\text {persons }}$.

\subsection{Experimental Results}

Table 1 shows recognition results for the new online learning algorithm (Bayesian SMC), compared with a batch learning algorithm (Bayesian MCMC), with which we estimated the predictive distribution of parameters using a Markov Chain Monte Carlo method[7]. Template images and test sequences showed 7 Japanese actors and 3 Japanese actresses in frontal pose against a blue background. For the template images, each individual shows a neutral facial expression, while for the test sequences, subjects were encouraged to show expressions and to talk freely. For the Bayesian SMC system, we used 18 sample images showing six fundamental expressions (happiness, sadness, fear, anger, disgust and surprise) to estimate the covariance matrix $\Lambda_{j}$. For the Bayesian MCMC system, we used the same data to draw $N_{j}$ samples of parameters. We set the number of Monte Carlo samples for each face model to $N_{j}=3600$ for both systems. We assumed that initial face regions were pre-detected so that the center position and radius of the face region were already available. Table 1 shows that the proposed approach reduced the ID error rate from $11.0 \%$ to $2.3 \%$, but that the total processing time was roughly double that of the original batch algorithm.

Table 1. Face recognition results (ID error rate)

\begin{tabular}{|c|c|c|}
\hline Model & Bayesian MCMC & Bayesian SMC \\
\hline ID error rate & $11.0 \%$ & $2.3 \%$ \\
\hline Processing time & 1155 sec. & 2329 sec. \\
\hline
\end{tabular}
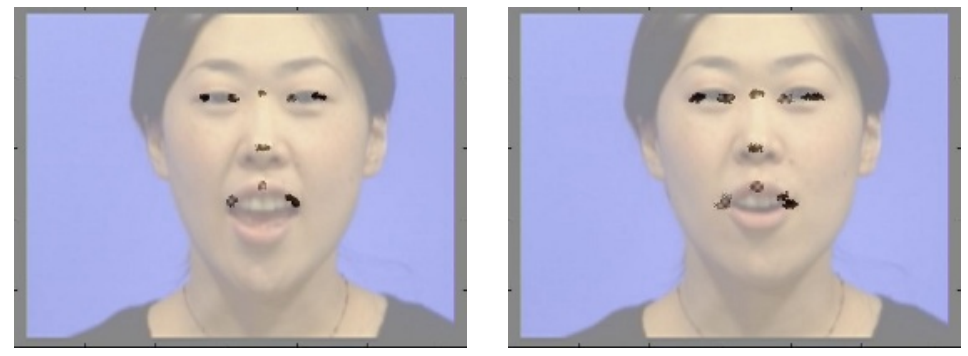

Fig. 1. Examples of SMC sample feature points at frame $n=0$ (left) and frame $n=8$ (right). The top 100 samples in order of the importance weights are shown.

Figure 1 shows examples of SMC particles (i.e. sets of feature point parameters) with the top 100 normalized importance weights out of a total of 36,000 particles. It is apparent that the proposed algorithm updates the posterior distribution of feature points more or less successfully despite facial deformations 
(reference feature points are located at the six eye and mouth corners plus the three locations on the mid-sagittal line).

\subsection{Pruned Resampling}

Figure 2 shows the evolution of the number of particles $N_{j}$ drawn by the Bayesian SMC model given a test video sequence of person $j_{\text {true }}=10$. The number of particles for $j=10$ grows steadily with time (frame number) while the others are either static or gradually decrease. The tendency for a particular model to accumulate ever larger numbers of particles at the expense of the other models reflects the increasing confidence of the system, with increasing volumes of data, that the given model is correct and the others incorrect.

However, much of the associated computation is unnecessary; we do not require so many particles to verify a well-supported hypothesis. Thus we introduce a further normalization into the resampling process such that the number of particles for the most likely model $\widehat{j}=\arg \max _{k} N_{k}=\arg \max _{k} P\left(y_{n} \mid y_{1: n-1}, \mathcal{H}_{k}\right)$, rather than the total number $N_{\text {total }}=\sum_{j} N_{j}$, remains approximately constant. Since the resampling process shares out the total mass of normalized importance weights at the previous step, it is natural to set the new total number of particles $N_{\text {total }}$ as follows:

$$
N_{\text {total }}=N_{\widehat{j}} \times \frac{\left.\sum_{k=1}^{N_{\text {persons }}} \sum_{i=1}^{N_{k}} \tilde{w}_{n}^{(i)}\right|_{\mathcal{H}_{k}}}{\left.\sum_{i=1}^{N_{\hat{j}}} \tilde{w}_{n}^{(i)}\right|_{\mathcal{H}_{\hat{j}}}}=N_{\widehat{j}} \times \frac{\sum_{k=1}^{N_{\text {persons }}} P\left(y_{n} \mid y_{1: n-1}, \mathcal{H}_{k}\right)}{P\left(y_{n} \mid y_{1: n-1}, \mathcal{H}_{\hat{j}}\right)}
$$

This "pruned resampling" maintains nearly constant the number of particles (and hence the volume of computation) at each step associated with the most likely model. In so doing, it reduces the amount of attention paid to increasingly unlikely models faster than does the original resampling scheme.

Table 2 shows recognition results with the pruned resampling scheme. Also shown is the total number of particles in existence $\bar{N}_{\text {total }}$, averaged over the 10 input video test sequences and 30 frames per sequence. Figure 3 shows the evolution of the number of particles $N_{j}$ using the pruned resampling scheme, given the same test data used in Figure $2\left(j_{\text {true }}=10\right)$. Table 2 and Figure 3 show that the new resampling approach successfully prunes redundant particles and computation without introducing any new identification errors. Total processing time is comparable to that of batch MCMC, but the significantly better recognition performance of SMC is not sacrificed.

Table 2. Face recognition results (ID error rate) with and without pruned resampling

\begin{tabular}{|c|c|c|c|}
\hline Model & Bayesian MCMC & \multicolumn{2}{|c|}{ Bayesian SMC } \\
\hline Pruned resampling? & $\mathrm{n} / \mathrm{a}$ & no & yes \\
\hline ID error rate & $11.0 \%$ & $2.3 \%$ & $2.3 \%$ \\
\hline Processing time & $1155 \mathrm{sec}$. & 2329 sec. & 1135 sec. \\
\hline $\bar{N}_{\text {total }}$ & 36000 & 36000 & 17488 \\
\hline
\end{tabular}


$\mathrm{Nj}$ : number of particles

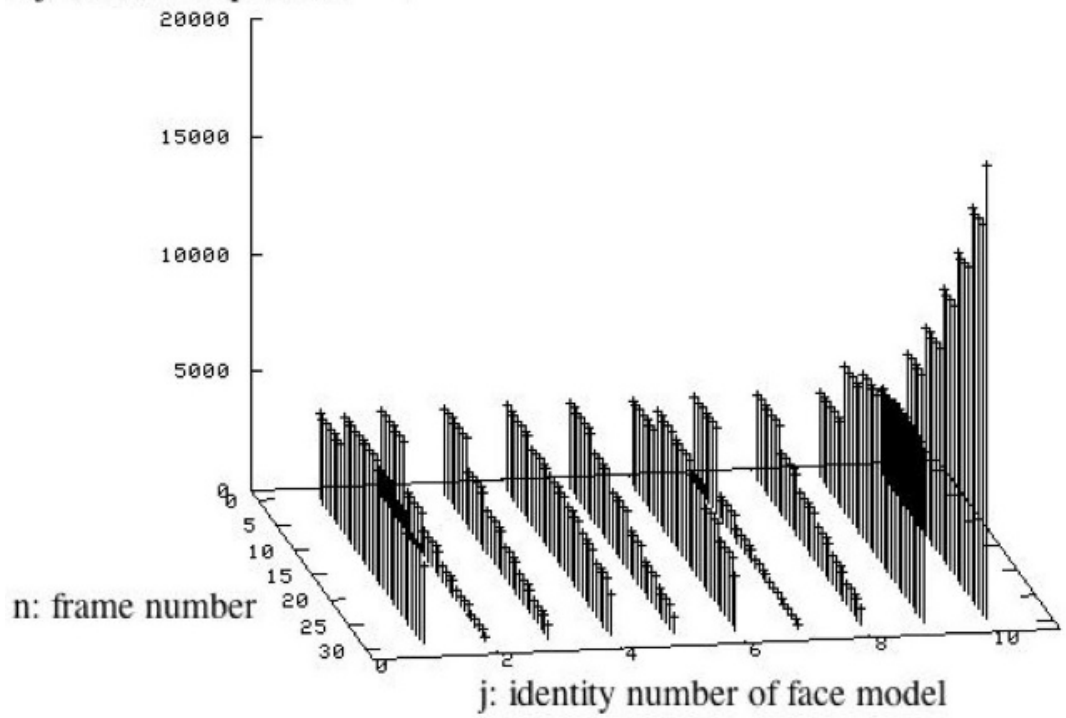

Fig. 2. Evolution of the number of SMC particles $N_{j}$ with ordinary resampling $\left(j_{\text {true }}=10, N_{\text {total }}=N_{1}+N_{2}+\ldots+N_{10}=36,000\right)$

$\mathrm{Nj}$ : number of particles

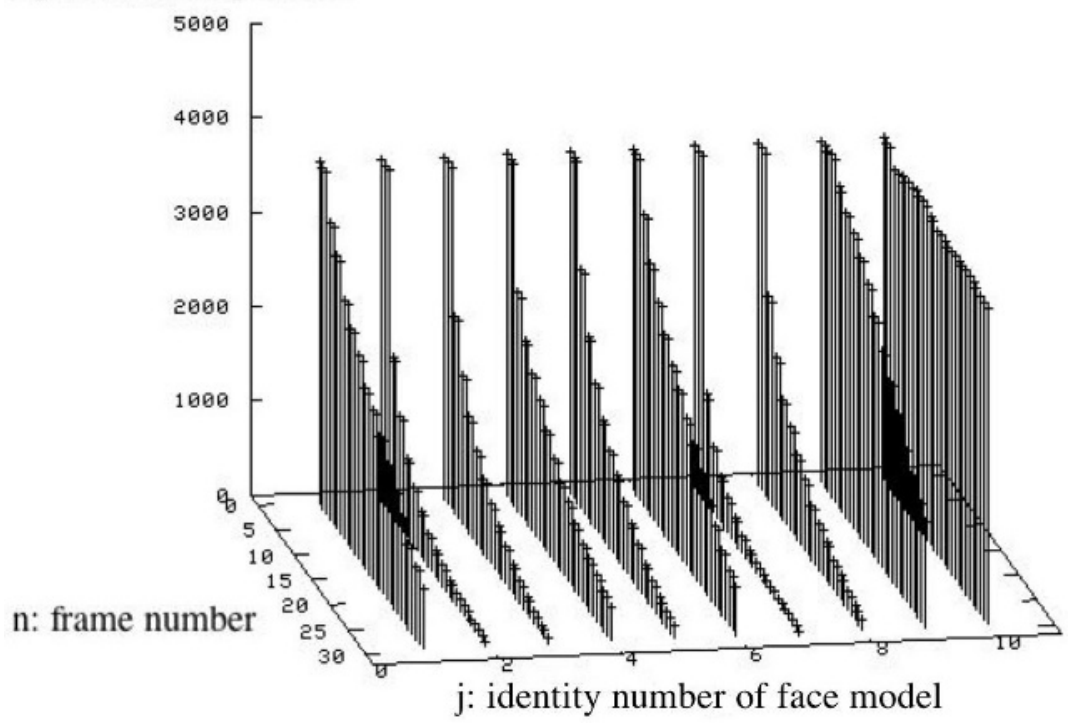

Fig. 3. Evolution of the number of SMC particles $N_{j}$ with pruned resampling $\left(j_{\text {true }}=10, N_{\widehat{j}}=N_{10} \cong 3,600\right)$ 


\section{Conclusions}

We introduced a new Sequential Monte Carlo (SMC) algorithm for online Bayesian learning in the context of a face recognition system based on deformable template matching. The proposed algorithm achieves markedly superior robustness of recognition against facial deformations by comparison to a baseline batch MCMC algorithm. A modification to the resampling stage of the new algorithm restores its computational cost to less than that of the baseline MCMC algorithm without sacrificing any of the gain in recognition performance.

Topics remaining for further work include the automation of the face detection stage and its combination with the SMC algorithm, and extensions to deal with larger image motions and changes in face pose and lighting conditions. The SMC based change detection algorithm described in [10 may be useful in this regard.

\section{References}

1. Clippingdale, S., Ito, T.: A Unified Approach to Video Face Detection, Tracking and Recognition. Proc. ICIP'99, Kobe, Japan (1999) 662-666

2. Clippingdale, S., Ito, T.; Partial automation of database acquisition in the FAVRET face tracking and recognition system using a bootstrap approach. Proc. MVA2000, Tokyo, Japan, (2000) 5-8

3. Wiskott, L., Fellous, J. M., Krüger, N., von der Malsburg, C.: Face Recognition by Elastic Bunch Graph Matching. TR96-08, Institut für Neuroinformatik, RuhrUniversitat Bochum (1996)

4. Doucet, A.: On Sequential Simulation-Based Methods for Bayesian Filtering. Technical report CUED/F-INFENG/TR-310, Cambridge University (1998)

5. Liu, J. S.: Monte Carlo Strategies in Scientific Computing. Springer, New York (2001) 53-77

6. Andrieu, C., Freitas, C. N., Doucet, A., Jordan, M. I.: An Introduction to MCMC for Machine Learning. Machine Learning, 50 (2003) 5-43

7. Matsui, A., Clippingdale, S., Uzawa F., Matsumoto, T.: Bayesian Face Recognition using a Markov Chain Monte Carlo Method. Proc. ICPR2004, 3 (2004) 918-921

8. Mardia, K. V., Jupp, P.: Directional Statistics. John Wiley and Sons Ltd., 2nd edition (2000)

9. Mackay, D. J. C.: Information Theory, Inference, and Learning Algorithms. Cambridge University Press (2003)

10. Matsumoto, T.: Marginal Likelihood Change Detection: Particle Filter Approach. Proc. International Workshop on Bayesian Inference and Maximum Entropy for Science and Engineering, AIP Conf. Proc. 803 (2005) 129-136 